\title{
CMU Hammerheads 2001 Team Description
}

\author{
Steve Stancliff, Ravi Balasubramanian, Tucker Balch, Rosemary Emery, \\ Kevin Sikorski, and Ashley Stroupe \\ Robotics Institute, Carnegie Mellon University \\ Pittsburgh, PA, 15213 \\ \{stancliff, ravib, tucker.balch, rosemary.emery, k.sikorski, \\ ashley. stroupe\}@cmu.edu \\ http://www.cs.cmu.edu/ coral/minnow
}

\section{Introduction}

The CMU MultiRobot Lab focuses on the study of team behavior in dynamic and uncertain environments. In order to further this research, we have developed an inexpensive autonomous robot platform, the Minnow. The CMU Hammerhead robot soccer team competes in the middle-size RoboCup competition using four of these Minnow robots.

The challenges of robot soccer require the robot players both to be individually capable and also to work together with their teammates. Some of the research areas which have been furthered by our work in robot soccer include color vision, multiagent sensor fusion, cooperative behaviors, and communication in noisy low-bandwidth environments.

The 2001 CMU Hammerheads represent the evolution of the previous year's team [1, using largely the same hardware and software architecture. The major changes in hardware are increases in processor speed and memory, and a change to digital cameras. On the software side, most of the low-level behaviors from the 2000 team are retained, and the emphasis has been on refining high-level behaviors and improving coordination and teamwork.

This paper describes the hardware and software architecture of the Minnow platform used in the CMU Hammerhead robot soccer team. Section 2 describes the hardware components of the robot platform. The software and control architectures are presented in Section 3. Finally, our strategies for teamwork and coordination are presented in Section 4.

\section{Robot Hardware}

The CMU Hammerheads are based on the Minnow robot platform (Figure 1). The Minnow is an inexpensive, fully autonomous robot with wireless communication and color vision. The underlying mechanical platform is a commerciallyavailable Cye robot from Probotics. This platform was chosen for its low cost and highly accurate odometry. The platform consists of a two-wheeled drive unit and a passive trailer. The trailer provides ample area for mounting of hardware. The 


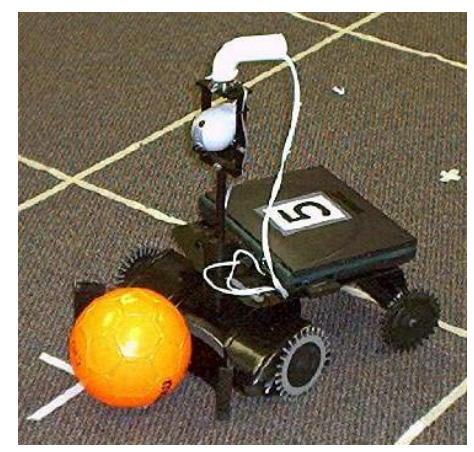

Fig. 1. Minnow robot platform.

drive unit includes a microcontroller which, in addition to controlling the motors, provides odometry and bump sensing. This microcontroller communicates with the host computer through an RS-232 connection.

The primary computing power of the Minnow is provided by a standard laptop computer mounted on the Cye trailer. A laptop was chosen over an embedded system in order to reduce platform development time and also for the convenience of having an onboard keyboard and video screen for calibration and debugging.

Vision is provided by a digital camera which is attached to the laptop computer through a USB port. The USB connection provides both signal and power; thus, the power for all computation and vision is provided by the laptop's batteries. The camera employs a wide-angle lens to allow the robot to see most of the playing field at once. The camera is mounted on a shaft above the drive unit, with a slipring for the electrical connections to the trailer, allowing the drive unit to rotate freely under the trailer.

Inter-robot communication is provided by wireless ethernet conforming to the IEEE 802.11 standard, consisting of PCMCIA cards on the robots and a wireless access point. In a noisy environment such as the RoboCup competition, the wireless access point provides a greater robot-to-robot communication range in comparison to a point-to-point network.

\section{Software}

\subsection{Vision Software}

The Minnows employ a color vision system as their primary means of information gathering. The camera images are processed by the CMVision software package [2] to detect colored regions (Figure 2). Because a wide-angle lens is used on the camera, the images must be dewarped to remove the spatial distortion caused by the lens [3]. CMVision is able to achieve high framerates by dewarping only the regions of interest rather than the entire image. 

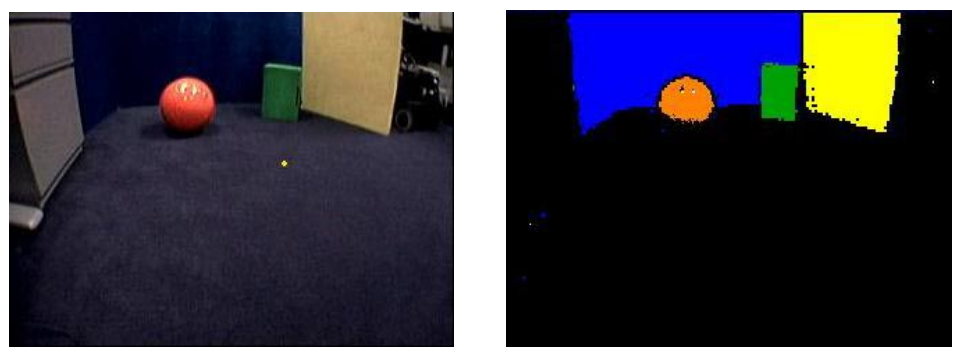

Fig. 2. An image before and after processing with CMVision.

\subsection{Control Software}

The robots are controlled using the Clay [4] architecture of TeamBots [5]. TeamBots is a Java-based package for the simulation and control of multiagent systems. The strength of TeamBots is that it allows for prototyping in simulation of the same control software that is used on the robots. The simulation environment (Figure 3) allows for the interaction of multiple robots, each running a different control system. This flexibility allows for the rapid prototyping of control schemes and allows for the early development of software in parallel with hardware.

Clay is a group of Java classes that can be easily combined into motor-schema based control systems, generally represented by finite-state machines. In this approach, each state corresponds to a suite of activated behaviors for accomplishing a task [6. Transitions between states are initiated by real-time perception. The activated behaviors create vectors to represent the desired trajectory of the robot. Clay is therefore similar to potential field navigation. It differs, however, in that only the vector at the robot's current position is calculated, rather than the entire vector field.

One advantage of the behavior-based approach for the Minnow robot is that it allows the control system to quickly locate and track the ball without having to explicitly deal with the position of the trailer. A planning approach would require the control system to plan with non-holonomic constraints, which is computationally difficult and time-consuming and thus inappropriate for a highly dynamic environment such as soccer.

\section{Teamwork and Coordination}

The CMU Hammerheads accomplish teamwork primarily through the sharing of information. The robots communicate to each other the location of the ball and their current operating state.

Communication of ball position between the robots improves the performance of the team by increasing the effective sensor range of individual robots and also by increasing the accuracy of ball-position estimates. The global ball-position 


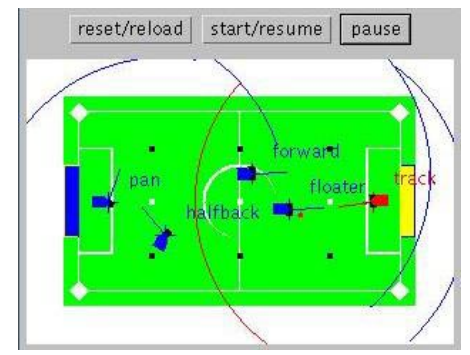

Fig. 3. TeamBots simulation.

estimate is used to guide a robot which does not see the ball so that it does not have to perform a blind search for the ball. The location of the ball is represented probabilistically, with each robot's ball-position estimate represented as a Gaussian. The Gaussians produced by the different robots are multiplied to provide the global estimate of the ball position [7].

Communication of player-state information is used to enable the robots to better coordinate their actions. For instance, when the goalie leaves the goal box, the fullback will position itself in front of the goal. Also, the two offensive players will switch roles dynamically depending on the location of the ball and which team has possession of it.

\section{References}

[1] Rosemary Emery, Tucker Balch, Rande Shern, Kevin Sikorski, and Ashley Stroupe. $\mathrm{Cmu}$ hammerheads team description. In Tucker Balch, Peter Stone, and Gerhard Kraetzschmar, editors, Proceedings of the 4th International Workshop on RoboCup, Melbourne, Australia, 2000.

[2] Jim Bruce, Tucker Balch, and Manuela Veloso. Fast and cheap color vision on commodity hardware. In Workshop on Interactive Robotics and Entertainment, pages 11-15, Pittsburgh, PA, April 2000.

[3] Hans Moravec. Robot spatial perception by stereoscopic vision and 3D evidence grids. Technical Report CMU-RI-TR-96-34, Robotics Institute, Carnegie Mellon University, September 1996.

[4] Tucker Balch. Clay: Integrating motor schemas and reinforcement learning. Technical Report GIT-CC-97-11, College of Computing, Georgia Institute of Technology, March 1997.

[5] Tucker Balch. Behavioral Diversity in Learning Robot Teams. PhD thesis, College of Computing, Georgia Institute of Technology, December 1998.

[6] Ronald Arkin and Douglas MacKenzie. Temporal coordination of perceptual algorithms for mobile robot navigation. IEEE Transactions on Robotics and Automation, 10(33):276-286, June 1994.

[7] A. Stroupe, M.C. Martin, and T. Balch. Merging probabilistic observations for mobile distributed sensing. Technical Report CMU-RI-TR-00-30, Robotics Institute, Carnegie Mellon University, December 2000. 